\title{
Hemodynamic and prognostic impact of the diastolic pulmonary arterial pressure in children with pulmonary arterial hypertension-a registry-based analysis
}

\author{
Christian Apitz ${ }^{1,2}$, Rolf M. F. Berger ${ }^{3}$, D. Dunbar Ivy ${ }^{4}$, Tilman Humpl ${ }^{5}$, Damien Bonnet ${ }^{6}$, \\ Maurice Beghetti ${ }^{7}$, Dietmar Schranz ${ }^{2}$, Heiner Latus ${ }^{2,8}$; on behalf of the TOPP investigators \\ ${ }^{1}$ Division of Pediatric Cardiology, University Children's Hospital Ulm, Ulm, Germany; ${ }^{2}$ Pediatric Heart Center, University Children's Hospital \\ Frankfurt/Giessen, Frankfurt, Germany; ${ }^{3}$ Center for Congenital Heart Diseases, Pediatric Cardiology, Beatrix Children's Hospital, University \\ Medical Center Groningen, University of Groningen, Groningen, the Netherlands; ${ }^{4}$ Pediatric Cardiology, Children's Hospital Colorado, University \\ of Colorado School of Medicine, Aurora, CO, USA; ${ }^{5}$ Division of Neonatology and Critical Care Medicine, University Children's Hospital Bern, \\ Bern, Switzerland; ${ }^{6}$ Centre de Référence Malformations Cardiaques Congénitales Complexes, M3C-Necker Hospital for Sick Children, Assistance \\ Publique des Hôpitaux de Paris, Pediatric Cardiology, University de Paris, Paris, France; ${ }^{7}$ Pediatric Cardiology Unit, Children's University Hospital, \\ Centre Universitaire Romand de Cardiologie et Chirurgie Cardiaque Pédiatrique, University of Geneva and Lausanne, Geneva, Switzerland; \\ ${ }^{8}$ Department of Pediatric Cardiology and Congenital Heart Disease, Deutsches Herzzentrum München, Technische Universität München, \\ München, Germany \\ Contributions: (I) Conception and design: C Apitz, D Schranz, H Latus, DD Ivy, RMF Berger; (II) Administrative support: None; (III) Provision \\ of study materials or patients: All authors; (IV) Collection and assembly of data: All authors; (V) Data analysis and interpretation: All authors; (VI) \\ Manuscript writing: All authors; (VII) Final approval of manuscript: All authors. \\ Correspondence to: Prof. Christian Apitz, MD. Division of Pediatric Cardiology, University Children's Hospital Ulm, Eythstr. 24, D-89075 Ulm, \\ Germany. Email: capitz@aol.com.
}

Background: Diastolic pulmonary arterial pressure (dPAP) is regarded to be less sensitive to flow metrics as compared to mean PAP (mPAP), and was therefore proposed for the assessment of a precapillary component in patients with postcapillary pulmonary hypertension $(\mathrm{PH})$. To analyze the diagnostic and prognostic impact of $\mathrm{dPAP}$ in patients with pure precapillary $\mathrm{PH}$, we purposed to compare the correlation between $\mathrm{dPAP}$ and mPAP, as well as hemodynamically-derived calculations [ratio of PAP to systemic arterial pressure (PAP/SAP), pulmonary vascular resistance index (PVRI), transpulmonary gradient (TPG)], using both dPAP and mPAP, at rest and during acute vasoreactivity testing (AVT) in children with idiopathic or heritable pulmonary arterial hypertension (IPAH/HPAH). Furthermore, we aimed to assess the association of these metrics (at baseline and changes after AVT) with transplant-free survival.

Methods: We conducted a retrospective analysis of the TOPP (Tracking Outcomes and Practice in Pediatric Pulmonary Hypertension) registry including 246 IPAH/HPAH patients. Of these, 45 children (18.3\%) died, and 13 (5.3\%) received lung transplantation during the observation period.

Results: dPAP and mPAP-derived variables showed almost linear relationship. Higher mPAP/mSAP, and dPAP-/mPAP-derived PVRI at rest was associated with time to death/transplantation. At maximum AVTresponse, the decrease of $\mathrm{dPAP}$ and $\mathrm{mPAP}$, diastolic pulmonary gradient (DPG) and TPG, as well as dPAP/ $\mathrm{dSAP}$ and $\mathrm{mPAP} / \mathrm{mSAP}$ was associated with time to death/transplantation, showing higher significance than corresponding baseline values. Remarkably, no predictive value was found for PVRI-reduction during AVT, neither dPAP- nor mPAP-derived.

Conclusions: There is a strong relationship between $\mathrm{APAP}$ and $\mathrm{mPAP}$-derived variables. According to our results, hemodynamics during AVT (irrespectively of dPAP- or mPAP-derived) may have more prognostic implications than resting hemodynamics in children with IPAH/HPAH, except for PVRI.

Keywords: Pulmonary arterial hypertension (PAH); acute vasoreactivity testing (AVT); cardiac catheterization; pulmonary arterial pressure (PAP); pulmonary vascular resistance; pediatric cardiology 
Submitted Nov 14, 2020. Accepted for publication Dec 23, 2020.

doi: $10.21037 / \mathrm{cdt}-20-934$

View this article at: http://dx.doi.org/10.21037/cdt-20-934

\section{Introduction}

Idiopathic or heritable pulmonary arterial hypertension (IPAH/HPAH) is a life-threatening disease caused by pathologic remodeling of the pulmonary arteries leading to an increase of pulmonary vascular resistance, and as a consequence also pulmonary arterial pressure (PAP), a condition that might potentially result in right ventricular failure, and eventually death $(1,2)$.

Since PAH is usually defined by the value of mean PAP (mPAP) and (mPAP-derived) pulmonary vascular resistance index $\{\mathrm{PVRI}=[\mathrm{mPAP}-$ pulmonary arterial wedge pressure (PAWP)]/pulmonary blood flow $(3,4)$, invasive assessment by cardiac catheterization is an essential part of diagnostic work-up in children with PAH (5). Furthermore, the invasively assessed hemodynamic variables and its changes during acute vasoreactivity testing (AVT) serve for risk stratification in pediatric IPAH/HPAH (6).

Cardiac catheterization is usually performed according to systematic protocols and includes amongst other parameters systolic, mean and diastolic systemic arterial, as well as systolic PAP, mPAP and diastolic PAP (dPAP), and the PAWP. In addition, invasive hemodynamic assessment includes calculation or measurement of cardiac output (CO) and quantification of PVRI, and the transpulmonary gradient $(\mathrm{TPG}=\mathrm{mPAP}-\mathrm{mPAWP})$. In both, children and adults with $\mathrm{PAH}$, these hemodynamic values are used to grade the severity of adverse pulmonary vascular remodelling and have shown to be associated with the patients' clinical status, right ventricular dysfunction and outcome (7-9).

Which of the assessed parameters during AVT is superior to predict favorable outcome has not been systematically studied in children so far. According to current recommendations, mPAP (derived from the area under the pressure curve and defined as the pressure level that divides the area under the pressure curve into two equal parts) is generally used for the definition of PAH, estimation of disease severity and the identification of vasoreactive response (in addition to $\mathrm{CO}$ and/or PVR depending of the definition). However, potential drawback of mPAP might be the dependency on CO and pulmonary arterial compliance, as has been postulated by studies in patients with postcapillary pulmonary hypertension $(\mathrm{PH})$ $(10,11)$. Naeije et al. as well as Gerges et al. were able to demonstrate that dPAP may be less sensitive to flow metrics as compared to $\mathrm{mPAP}$, which was the rationale in the past of proposing diastolic pulmonary gradient $(\mathrm{DPG}=$ dPAP - mPAWP) rather than TPG for the assessment of a precapillary component in patients with group $2 \mathrm{PH}(10,11)$.

While the interest around the utility of dPAP and DPG as a hemodynamic marker has been primarily centered on the phenotype of $\mathrm{PH}$ due to left heart disease, it could also be of interest to see if there is a major difference in hemodynamic derivations from $\mathrm{dPAP}$ and $\mathrm{mPAP}$ in patients with pure precapillary $\mathrm{PH}$ (12).

Accordingly, we conducted a registry-based analysis of the international TOPP (Tracking Outcomes and Practice in Pediatric Pulmonary Hypertension) registry in order to compare the correlation between $\mathrm{APAP}$ and mPAP, as well as hemodynamically-derived calculations [ratio of PAP to systemic arterial pressure (PAP/SAP), PVRI, TPG], using both $\mathrm{dPAP}$ and mPAP, at rest and during AVT. In addition, we aimed to assess the association of these metrics (at baseline and changes after AVT) with transplant-free survival.

We present the following article in accordance with the MDAR reporting checklist (available at http://dx.doi. org/10.21037/cdt-20-934).

\section{Methods}

TOPP is a center-based registry covering 31 centers in 19 countries, which was initiated on January 31st, 2008 (1). TOPP collects data on the assessment, treatment and followup of pediatric $\mathrm{PH}$ patients as performed by judgment of their treating physician. Participating centers include consecutive patients aged between 3 months and 18 years, presenting with $\mathrm{PAH}$ or with $\mathrm{PH}$ groups 3-5 (classified according to the 2003 3rd World Pulmonary Hypertension Symposium) and diagnosed on or after January 1st, 2001 (1). Patients were eligible for TOPP inclusion when meeting pre-specified hemodynamic criteria: $\mathrm{mPAP}$ of $\geq 25 \mathrm{mmHg}$, PVRI of $\geq$ $3 \mathrm{WU} \cdot \mathrm{m}^{2}$ and a mean PAWP of $\leq 12 \mathrm{mmHg}$.

The TOPP executive board members reviewed all cardiac catheterization data sets to confirm diagnosis and 
Table 1 Hemodynamic measurements at baseline and at maximum response as well as percentage reduction at maximum response from baseline during AVT

\begin{tabular}{|c|c|c|c|c|}
\hline Parameter & Baseline & Maximum response & Percentage reduction & $P$ value \\
\hline mPAP $(\mathrm{mmHg})$ & $58.2 \pm 17.9$ & $47.5 \pm 20.1$ & $18.9 \pm 19.4$ & $<0.001$ \\
\hline dPAP-derived PVRI (WU·m²) & $11.4 \pm 8.3$ & $8.4 \pm 8.4$ & $36.0 \pm 38.6$ & $<0.001$ \\
\hline mPAP-derived PVRI $\left(\mathrm{WU} \cdot \mathrm{m}^{2}\right)$ & $17.5 \pm 11.5$ & $13.4 \pm 11.0$ & $26.5 \pm 28.4$ & $<0.001$ \\
\hline $\mathrm{mPAP} / \mathrm{mSAP}$ & $0.87 \pm 0.28$ & $0.71 \pm 0.32$ & $20.1 \pm 21.9$ & $<0.001$ \\
\hline $\mathrm{DPG}(\mathrm{mmHg})$ & $32.6 \pm 16.1$ & $23.3 \pm 17.4$ & $34.5 \pm 37.3$ & $<0.001$ \\
\hline TPG $(\mathrm{mmHg})$ & $49.9 \pm 18.1$ & $38.7 \pm 20.4$ & $24.2 \pm 23.9$ & $<0.001$ \\
\hline
\end{tabular}

AVT, acute vasoreactivity testing; PAP, pulmonary arterial pressure; dPAP, diastolic PAP; mPAP, mean PAP; PVRI, pulmonary vascular resistance index; SAP, systemic arterial pressure; dSAP, diastolic SAP; mSAP, mean SAP; DPG, diastolic pulmonary gradient; TPG, transpulmonary gradient.

recalculated PVRI and CO by the Fick method using a single assumed oxygen consumption table (13) or the recorded $\mathrm{CO}$ as measured by thermodilution (if performed).

For the current study, data from the final data export in 2019 were analyzed.

Patients in whom no AVT was performed at diagnostic catheterization, or the administered vasodilator during AVT was considered inadequate (including $\mathrm{FiO}_{2}<0.45$ or unknown agents) were excluded from the analyses. Furthermore, patients with incomplete pressure or flow data at AVT were excluded.

The study was conducted in accordance with the Declaration of Helsinki (as revised in 2013). The protocol was reviewed and approved by the ethics committee of the Justus-Liebig-University Giessen (approval number 33/08) on behalf of the participating centers. Informed consent has been obtained from all patients and/or their legal guardians.

\section{Statistical analysis}

Data are presented as mean \pm standard deviation, or number (percentage) of patients. Statistical comparisons were made using $t$-test for normally distributed variables, MannWhitney $\mathrm{U}$ test for not-normally distributed variables, and Chi-Square test for categorical variables, as appropriate. Correlations were assessed by linear regression. Outcome analyses were based on transplant-free survival from hemodynamic evaluation, with death and transplantation as end-points. Patients without end-point were censored at last follow-up visit. Variables were split on their median for analyses with Kaplan-Meier curves. Subsequently, the correlation of continuous hemodynamic parameters with survival was evaluated by using Cox regression [hazard ratios (HRs)] for time to death/transplantation. A receiver operating characteristic (ROC) curve was used to identify the best threshold value (maximum sensitivity and specificity) for prediction of death/lung transplantation events. Analyses were performed using IBM SPSS statistics version 25.0 (IBM Inc., Armonk, NY, USA). The level of significance was defined as $\mathrm{P}<0.05$.

\section{Results}

Six hundred and eighty-six consecutive children with confirmed $\mathrm{PH}$ were enrolled in the registry. Three hundred and thirty-one children (48\%) were diagnosed with IPAH/ $\mathrm{HPAH}$ and were eligible for the study, 246 of them (mean age $7.6 \pm 5.1$ years, $63 \%$ female) with evaluable AVT (111 prevalent, 135 incident patients). The majority of patients (74.8\%) were in NYHA Functional class II and III, 10.6\% in class IV. Twenty-six patients (10.6\%) were classified as AVT responder according to Sitbon criteria $(14,15)$.

\section{Comparison $\mathrm{PPAP}$ versus $m P A P$}

Invasively assessed hemodynamic measurements at baseline and at maximum response as well as percentage decrease of these values at maximum response from baseline during AVT are presented in Table 1. Interestingly, dPAP variables showed smaller absolute reduction, but greater percentage 
Table 2 Regression analyses to compare the dependencies of dPAP- and mPAP-derived parameters (at baseline and at maximum response) on pulmonary flow, pulse pressure, and PAWP

\begin{tabular}{|c|c|c|c|}
\hline Parameter & Pulmonary flow & Pulse pressure & PAWP \\
\hline \multicolumn{4}{|l|}{ dPAP $(\mathrm{mmHg})$} \\
\hline Baseline & $-0.14(-0.27,-0.02)^{\star}$ & $0.31(0.19,0.42)^{\star \star \star}$ & $0.04(-0.08,0.17)$ \\
\hline Maximum response & $-0.17(-0.32,-0.00)^{\star}$ & $0.44(0.33,0.54)^{\star \star \star}$ & $0.07(-0.06,0.20)$ \\
\hline \multicolumn{4}{|l|}{ mPAP $(\mathrm{mmHg})$} \\
\hline Maximum response & $-0.13(-0.28,0.03)$ & $0.63(0.54,0.70)^{\star \star \star}$ & $0.02(-0.11,0.16)$ \\
\hline \multicolumn{4}{|c|}{ dPAP-derived PVRI $\left(\mathrm{WU} \cdot \mathrm{m}^{2}\right)$} \\
\hline Baseline & $-0.58(-0.66,-0.49)^{\star \star *}$ & $0.33(0.22,0.44)^{\star \star \star}$ & $-0.04(-0.16,0.09)$ \\
\hline Maximum response & $-0.47(-0.59,-0.33)^{\star \star \star}$ & $0.44(0.29,0.56)^{\star \star \star}$ & $-0.05(-0.22,0.11)$ \\
\hline Maximum response & $-0.53(-0.64,-0.39)^{\star \star *}$ & $0.48(0.35,0.60)^{\star \star \star}$ & $-0.01(-0.18,0.15)$ \\
\hline \multicolumn{4}{|l|}{ dPAP/dSAP } \\
\hline Baseline & $-0.03(-0.16,0.09)$ & $0.19(0.07,0.31)^{\star}$ & $0.02(-0.11,0.14)$ \\
\hline Maximum response & $-0.09(-0.25,0.07)$ & $0.39(0.27,0.49)^{\star \star \star}$ & $-0.02(-0.16,0.11)$ \\
\hline \multicolumn{4}{|l|}{$\mathrm{mPAP} / \mathrm{mSAP}$} \\
\hline Baseline & $-0.06(-0.18,0.07)$ & $0.35(0.24,0.46)^{\star \star \star}$ & $0.01(-0.11,0.14)$ \\
\hline Maximum response & $-0.08(-0.24,0.08)$ & $0.52(0.42,0.61)^{\star \star \star}$ & $-0.07(-0.20,0.07)$ \\
\hline \multicolumn{4}{|l|}{$\mathrm{DPG}(\mathrm{mmHg})$} \\
\hline
\end{tabular}

Data are presented as correlation coefficient $(95 \% \mathrm{Cl}) .{ }^{*}, \mathrm{P}<0.05 ;{ }^{* * *}, \mathrm{P}<0.001$. PAP, pulmonary arterial pressure; dPAP, diastolic PAP; mPAP, mean PAP; PAWP, pulmonary arterial wedge pressure; PVRI, pulmonary vascular resistance index; SAP, systemic arterial pressure; dSAP, diastolic SAP; mSAP, mean SAP; DPG, diastolic pulmonary gradient; TPG, transpulmonary gradient.

reduction. This is most relevant for the relative PVRI decrease at maximum response from baseline during AVT, which shows approximately $10 \%$ more reduction when calculated by dPAP. Compared with PVRI reduction, for the relative decrease of PAP/SAP ratios, the difference was remarkably minor between $\mathrm{DPAP}$ and mPAP derived ratios. Percentage reduction during AVT of dPAP vs. mPAP $(\mathrm{r}=0.90, \mathrm{P}<0.001)$, dPAP-derived PVRI $v s . \mathrm{mPAP}$-derived PVRI $(\mathrm{r}=0.86, \mathrm{P}<0.001)$, and $\mathrm{dPAP} / \mathrm{dSAP}$ vs. $\mathrm{mPAP} / \mathrm{mSAP}$ $(\mathrm{r}=0.89, \mathrm{P}<0.001)$ showed strong correlations.

\section{Correlations with pulmonary flow, pulse pressure, and PAWP}

Regression analyses demonstrated a negative correlation between dPAP- and mPAP-derived PVRI and pulmonary flow, which appears to be stronger for mPAP-derived PVRI (Table 2). Remarkably, there was a correlation between most dPAP- and mPAP variables and pulse pressure, showing stronger correlation for mPAP-derived parameters. No correlation was found between $\mathrm{dPAP}$ - or mPAP-variables 
Table 3 Impact of dPAP- and mPAP-derived parameters at BL and its changes (AR and PR) at maximum response from baseline during AVT using cox regression (HRs) for time to death/transplantation (adjusted for age and gender)

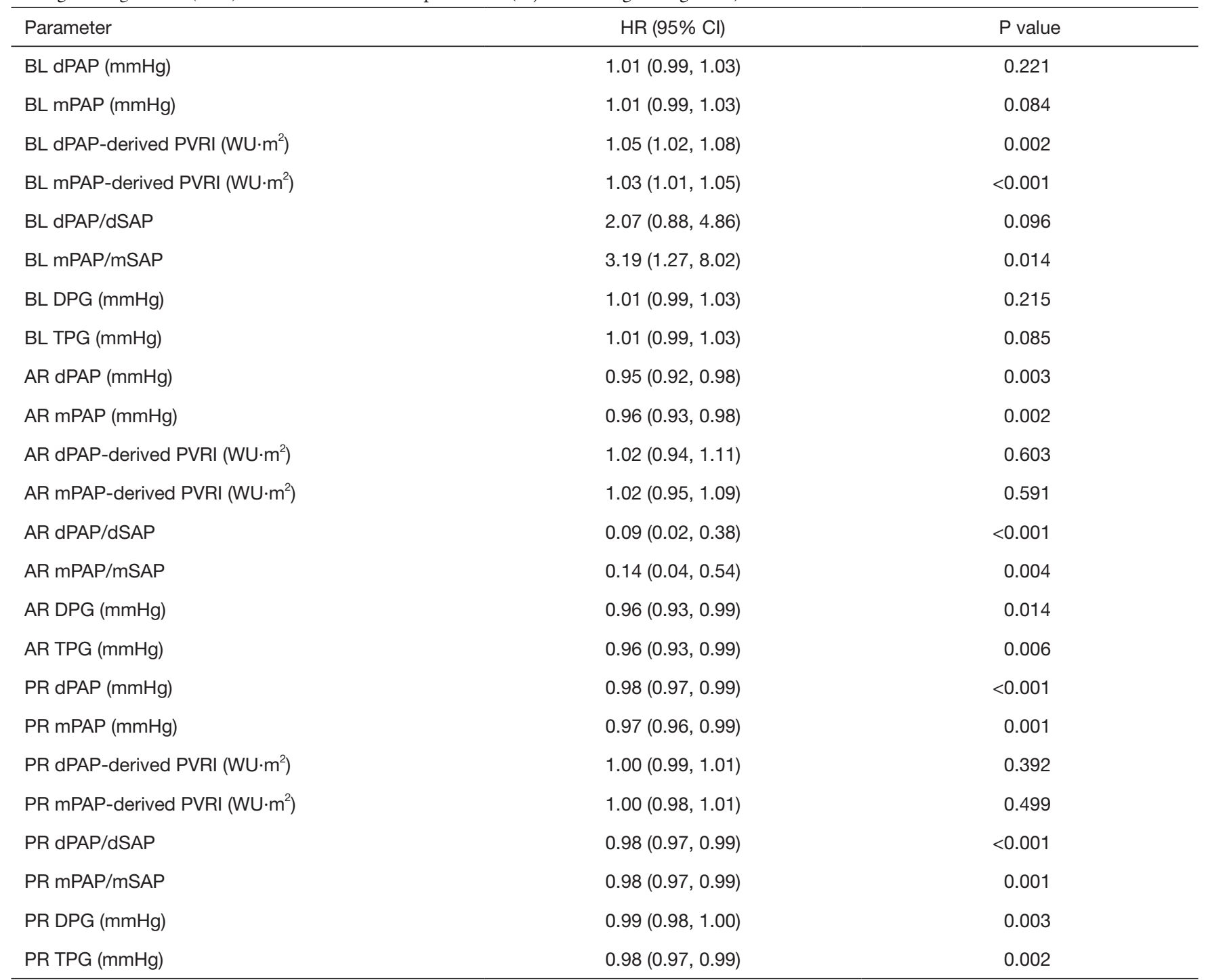

PAP, pulmonary arterial pressure; dPAP, diastolic PAP; mPAP, mean PAP; BL, baseline; AR, absolute reduction; PR, percentage reduction; AVT, acute vasoreactivity testing; HR, hazard ratio; PVRI, pulmonary vascular resistance index; SAP, systemic arterial pressure; dSAP, diastolic SAP; mSAP, mean SAP; DPG, diastolic pulmonary gradient; TPG, transpulmonary gradient.

and PAWP.

\section{Impact on disease prognosis}

During the observation period, 45 children (18.3\%) died, and $13(5.3 \%)$ received lung transplantation. Mean time from inclusion to death was $1.66 \pm 1.7$ years, mean time from inclusion to transplantation was $3.34 \pm 2.1$ years.

At baseline, neither dPAP, mPAP, nor dPAP/dSAP were significantly predictive for death/transplantation. However, higher baseline $\mathrm{mPAP} / \mathrm{mSAP}$, and higher baseline $\mathrm{dPAP}$ and mPAP-derived PVRI were associated with time to death/transplantation (Table 3).

At maximum response, absolute and percentage decrease of dPAP and mPAP, DPG and TPG, as well as reduction of $\mathrm{dPAP} / \mathrm{dSAP}$ and $\mathrm{mPAP} / \mathrm{mSAP}$ were associated with time to death/transplantation, showing higher significance than corresponding baseline values (Table 3).

Remarkably, no predictive value was found for absolute/ percentage reduction of PVRI during AVT, neither dPAP- 
nor mPAP-derived (Table 3).

Kaplan-Meier analysis of all four variables using the median as cut-off value revealed percentage reduction of PAP/SAP (irrespective of APAP or mPAP-derived) as best predictor for death/transplantation events [HR: 0.98 (95\% CI: 0.97, 0.99); $\mathrm{P}<0.001$; after adjustment for age and gender) (Figure 1). ROC curve revealed a PAP/SAP reduction of $20.4 \%$ as best cut-off [area under the curve 0.65 (95\% CI: 0.57, 0.73)] (Figure 2).

\section{Discussion}

This study adds a number of new insights into hemodynamic assessment of children with IPAH/HPAH and its prognostic implications. First, dPAP and dPAPderived parameters provided comparable hemodynamic information regarding severity of pulmonary vascular disease as mPAP and mPAP-derived parameters, however there was no obvious benefit for prognostic assessment of IPAH patients. Second, the absolute/percentage reduction of invasive hemodynamic parameters from baseline to maximum response during AVT appeared to carry high predictive value. Third, the ratio of PAP/SAP demonstrated to be the most consistent prognostic hemodynamic variable in pediatric IPAH/HPAH. Fourth, while PVRI at rest was related with outcome, reduction of PVRI during AVT (irrespectively of dPAP- or mPAP-derived) showed no predictive value.

\section{Comparison dPAP versus $m P A P$}

Since the 1st World Symposium on Pulmonary Hypertension (WSPH) in 1973, PH has been defined by the mPAP assessed by right heart catheterization (3). The dPAP is generally additionally measured, but was mostly disregarded for hemodynamic analysis in $\mathrm{PH}$-patients for a long period of time.

Our data demonstrated dPAP and dPAP-derived variables being useful parameters for hemodynamic assessment of pulmonary vascular disease, similar reliable as mPAP and mPAP-derived parameters. In accordance with these findings, there were strong correlations between its responses to AVT. However, our results also highlighted, that for the data interpretation the specific characteristics of dPAP need to be considered, particularly the observed feature that dPAP variables showed smaller absolute reduction, but greater percentage decrease during AVT compared to mPAP parameters. This appeared to be most relevant for the PVRI reduction at maximum response from baseline during AVT, which showed approximately $10 \%$ more decrease when calculated by dPAP. Compared to PVRI, PAP/SAP ratio showed a percentage reduction during AVT that was more similar for both, dPAP and mPAP, and might therefore serve as a more robust parameter for the interpretation of AVT-response.

\section{Correlations with pulmonary flow, pulse pressure, and PAWP}

Increased right ventricle (RV) afterload resulting in RV failure is the main reason of mortality in PAH. RV afterload consists of static components (PVR and mPAP) which define the net forward flow, and pulsatile components responsible of flow and pressure oscillations. Pulsatile components include pulmonary arterial compliance, the ability of pulmonary arteries to accommodate oscillatory changes in volume, and pulmonary stroke volume, the amount of blood ejected in the pulmonary artery per cardiac cycle. Conventional hemodynamic parameters such as mPAP and PVRI only partially represent RV afterload, since they reflect only its static components and not its pulsatile components. In healthy subjects the pulsatile components contribute little to the RV afterload, due to the low resistance and high compliance of the pulmonary circulation. However in PAH patients their contribution to $\mathrm{RV}$ afterload becomes increasingly important due to arterial stiffening and increased reflection waves $(16,17)$.

In our study, we assessed pulmonary flow and pulmonary pulse pressure, but unfortunately not heart rate to determine pulmonary stroke volume. Therefore, we were not able to calculate pulmonary arterial compliance. However, the correlations of our hemodynamic variables with pulmonary pulse pressure suggests a remarkable relationship. Our results did not detect significant differences of dPAPderived compared to $\mathrm{mPAP}$-derived variables in regard to dependencies on pulsatile components of $\mathrm{RV}$ afterload, i.e., pulmonary flow and pulmonary pulse pressure, as previously described in adult patients with postcapillary $\mathrm{PH}$ (10).

\section{Impact on disease prognosis}

Prognostic parameters are used in $\mathrm{PAH}$ patients to guide medical treatment. Hemodynamic prognostic variables recommended by current guidelines include only baseline values at catheterization, i.e., right atrial pressure, systemic venous saturation, pulmonary vascular resistance and 

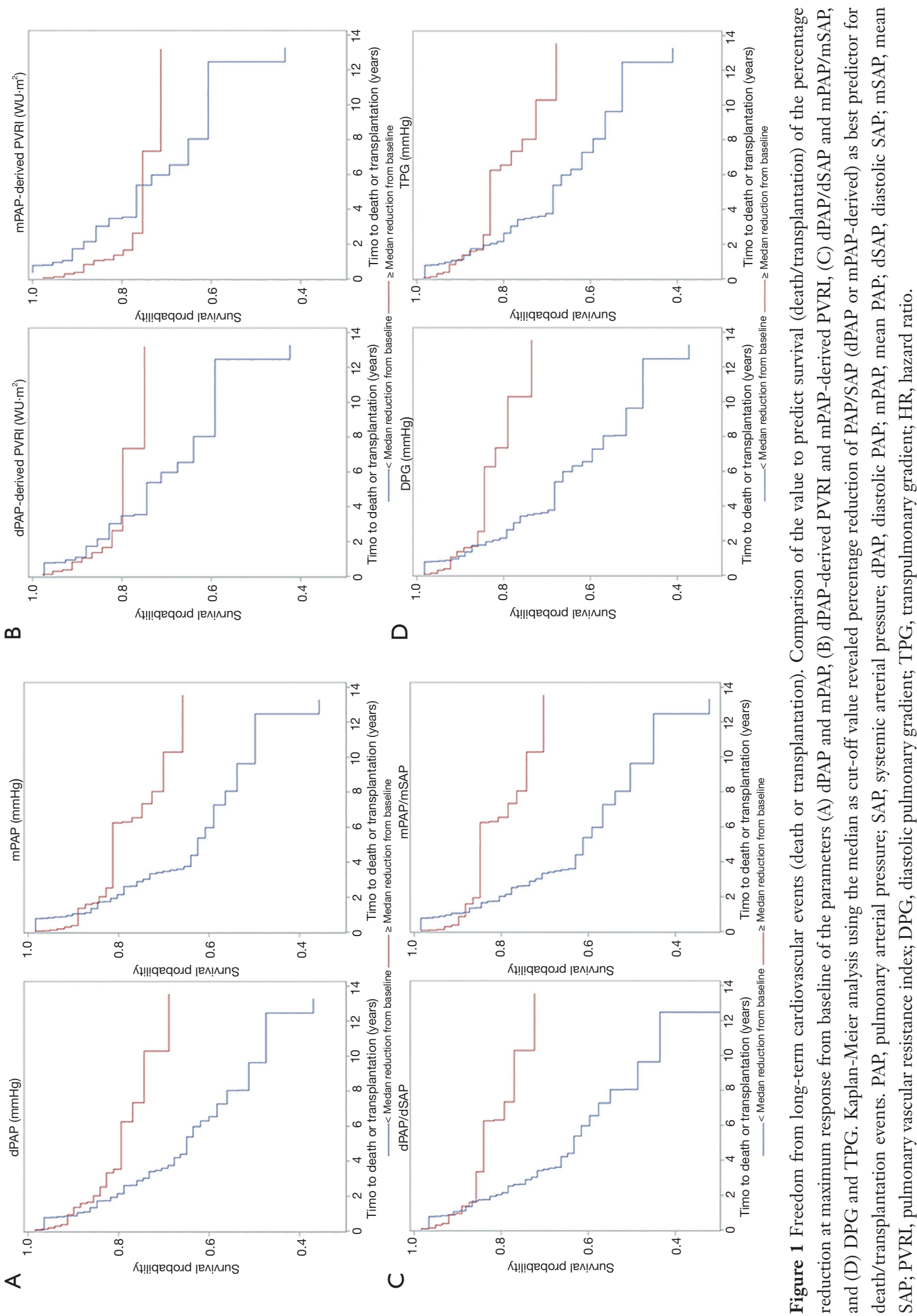

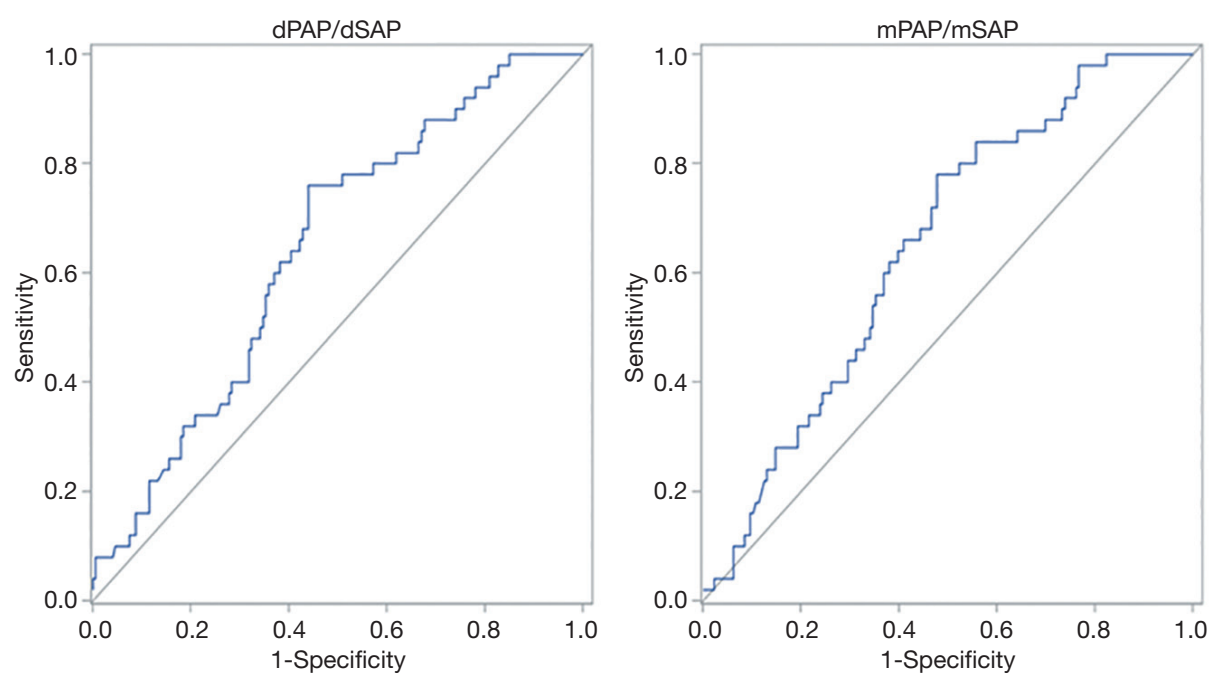

\begin{tabular}{ccc} 
& dPAP/dSAP & mPAP/mSAP \\
\hline Area under the curve (AUC) (95\% Cl) & $0.644(0.563,0.726)$ & $0.649(0.571,0.728)$ \\
Optimal cut-oft (Youden index) & 20.385 & 20.468 \\
Sensitvity at optimal cut-off & 0.760 & 0.780 \\
(1-Specifcity) at optimal cut-off & 0.439 & 0.477 \\
\hline
\end{tabular}

Figure 2 ROC curve for the assessment of best cut-off for PAP/SAP reduction to predict survival. ROC curve revealed a PAP/SAP reduction of $20.4 \%$ as best cut-off. ROC, receiver operating characteristic; PAP, pulmonary arterial pressure; SAP, systemic arterial pressure; dPAP, diastolic PAP; mPAP, mean PAP; dSAP, diastolic SAP; mSAP, mean SAP.

cardiac index (4). Positive response during AVT is also suggested as predictor, however is not well defined in children (15). Furthermore, changes in hemodynamic parameters have not previously been shown to be predictive of outcome (4).

Our data demonstrated that in addition to the baseline value, the reduction (absolute and percentage) of a number of hemodynamic parameters from baseline to maximum response during AVT showed remarkable predictive value. While it is known that patients with vasoreactive PAH have better prognosis, the definition of AVT response is still a matter of debate in children with IPAH/HPAH. Therefore, this study may help to expand the evidence on this topic. According to our results, the PAP/SAP ratio demonstrated to be the most consistent prognostic hemodynamic variable in pediatric IPAH/HPAH. This highlights the importance of the PAP/SAP ratio especially in children where the $\mathrm{DPAP}$ and MPAP always have to be interpreted in the context of the simultaneously recorded SAP, since the absolute values of SAP are clearly age dependent and thus, the ratio facilitates the interpretation of PAP values in children $(5,18)$.

\section{Prognostic information of PVRI}

Baseline PVRI is an established predictor of worse outcome in PAH patients reflecting the disease severity $(19,20)$, and consequently, high baseline PVRI was recommended as a tool for risk stratification by the pediatric task force of the latest WSPH recommendations (4).

According to our results it has to be considered that the absolute or percentage reduction of PVRI during AVT is inappropriate for prognostication of children with IPAH/ HPAH. This is in line with earlier observations of Rich et al. who addressed the difference with regard to outcome between "pressure responders" (who responded to AVT with significant decrease of mPAP and PVR) and exclusively "resistance responders" in adult IPAH (21). They postulated different levels of severity of pulmonary vascular disease as explanation for the different responses with exclusively "resistance responders" having more advanced pulmonary vascular disease. Subsequently, Sitbon et al. further defined that an absolute drop in PAP during AVT, and not a drop in PVR predicted outcome in adult IPAH patients on Calcium-channel-blocker-therapy (14). This was later confirmed also for pediatric PAH by Douwes et al. with 
data from the TOPP-cohort, who identified change in PAP (Sitbon criteria) and not PVR (Barst/Reveal criteria) predictive for outcome (15).

\section{Study limitations}

Several limitations of this study are worth considering. The TOPP registry is a prospective observational international disease registry with associated limitations. Because of the observational nature of the registry, no standardized AVTprotocol was prescribed, nor the use of predefined response criteria or treatment strategies. Baseline hemodynamic measurements and AVT tests were performed using different protocols, including variation in baseline conditions and used acute vasodilator agents. Hemodynamic values measured by each TOPP-investigator were reviewed by the executive board for completeness and plausibility but could not be reassessed due to unavailability of curve recordings. However, with its large sample size from a real-world population and its global generalizability, the TOPP-registry is unique in providing important insights into invasive diagnostic evaluation and outcome of children with PAH.

The derivation of PVRI by the use of $\mathrm{APAP}$ is a limitation from a physical viewpoint. The resistance of the pulmonary circulation is calculated in analogy to Ohm's law $(\mathrm{R}=\mathrm{V} / \mathrm{I})$ and requires the potential difference or voltage $(\mathrm{V})$ in the numerator of the equation. The voltage $(\mathrm{V})$ or potential difference between two points is directly proportional to the current (I). Translated to the pulmonary circulation, pulmonary vascular resistance equals the mean pressure difference across the pulmonary circulation $(\triangle \mathrm{P}=\mathrm{mPAP}-$ mPAWP; i.e., TPG) divided by CO. By substituting mPAP with $\mathrm{DPAP}$ in this equation, the numerator is changed from TPG to DPG. However, as mentioned above, the difference between two mean pressures is required for the calculation of PVR. Therefore, it is imprecise to refer to "(dPAP mPAWP)/CO" as "dPAP-derived PVR". This index rather represents a flow-corrected expression of DPG (DPG/CO).

Regression analysis to assess the influence of pulmonary flow and PAWP on the selected hemodynamic parameters might be interpreted carefully, since physiologically exact this can only be answered by assessing changes in these parameters at different stages of flow and PAWP in a single patient during one examination (for example during exercise testing). It has been previously demonstrated that if PAWP is directly transmitted to $\mathrm{dPAP}$, there is a disproportional increase in sPAP and mPAP depending on stroke volume. TPG increases, but the DPG is independent of both PAWP and stroke volume (10).

\section{Conclusions}

There is a strong relationship between $\mathrm{dPAP}$ and mPAPderived variables. $\mathrm{dPAP}$-derived parameters provided similar reliable hemodynamic information regarding severity and prognosis of pulmonary vascular disease as mPAP-derived parameters. In addition to the baseline values, absolute/ percentage reduction of invasively assessed hemodynamic parameters from baseline to maximum response during AVT appeared to carry high predictive value, except for PVRI. Of these parameters, percentage reduction of PAP/ SAP during AVT showed the greatest potential to expand the spectrum of predictors of outcome in children with IPAH/HPAH.

\section{Acknowledgments}

The authors would like to express their respect and gratitude to Robyn J. Barst, who deceased in 2013, for her inspiring and continuous work as a founding member of the Association for Pediatric Pulmonary Hypertension and Tracking Outcomes and Practice in Pediatric Pulmonary Hypertension (TOPP) registry.

Funding: None.

\section{Footnote}

Provenance and Peer Review: This article was commissioned by the editorial office, Cardiovascular Diagnosis and Therapy for the series "Pediatric Pulmonary Hypertension". The article has undergone external peer review.

Reporting Checklist: The authors have completed the MDAR reporting checklist. Available at http://dx.doi.org/10.21037/ cdt-20-934

Data Sharing Statement: Available at http://dx.doi. org/10.21037/cdt-20-934

Conflicts of Interest: All authors have completed the ICMJE uniform disclosure form (available at http://dx.doi. org/10.21037/cdt-20-934). The series "Pediatric Pulmonary Hypertension" was commissioned by the editorial office without any funding or sponsorship. Dr. CA served as the unpaid Guest Editor of the series. Dr. RMFB reports grants from Actelion, during the conduct of the study; other from 
Actelion, other from GSK, other from Lilly, outside the submitted work. Dr. DDI reports grants from Actelion, during the conduct of the study; other from Actelion, other from GSK, other from Lilly, other from Bayer, other from Gossamer Bio, other from Liquidia, from United Therapeutics, outside the submitted work. Dr. DB reports grants from Actelion, during the conduct of the study; personal fees from Actelion, personal fees from Bayer, personal fees from Lilly, outside the submitted work. Dr. $M B$ reports grants from Actelion, during the conduct of the study; personal fees from Actelion, personal fees from Bayer, personal fees from Lilly, personal fees from GSK, personal fees from Pfizer, personal fees from Orphacare, outside the submitted work. The authors have no other conflicts of interest to declare.

Ethical Statement: The authors are accountable for all aspects of the work in ensuring that questions related to the accuracy or integrity of any part of the work are appropriately investigated and resolved. The study was conducted in accordance with the Declaration of Helsinki (as revised in 2013). The protocol was reviewed and approved by the ethics committee of the Justus-Liebig-University Giessen (approval number 33/08) on behalf of the participating centers. Informed consent has been obtained from all patients and/or their legal guardians.

Open Access Statement: This is an Open Access article distributed in accordance with the Creative Commons Attribution-NonCommercial-NoDerivs 4.0 International License (CC BY-NC-ND 4.0), which permits the noncommercial replication and distribution of the article with the strict proviso that no changes or edits are made and the original work is properly cited (including links to both the formal publication through the relevant DOI and the license). See: https://creativecommons.org/licenses/by-nc-nd/4.0/.

\section{References}

1. Berger RM, Beghetti M, Humpl T, et al. Clinical features of paediatric pulmonary hypertension: a registry study. Lancet 2012;379:537-46.

2. Schermuly RT, Ghofrani HA, Wilkins MR, et al. Mechanisms of disease: pulmonary arterial hypertension. Nat Rev Cardiol 2011;8:443-55.

3. Simonneau G, Montani D, Celermajer DS, et al. Haemodynamic definitions and updated clinical classification of pulmonary hypertension. Eur Respir J
2019;53:1801913.

4. Rosenzweig EB, Abman SH, Adatia I, et al. Paediatric pulmonary arterial hypertension: updates on definition, classification, diagnostics and management. Eur Respir J 2019;53:1801916.

5. Apitz C, Hansmann G, Schranz D. Hemodynamic assessment and acute pulmonary vasoreactivity testing in the evaluation of children with pulmonary vascular disease. Expert consensus statement on the diagnosis and treatment of paediatric pulmonary hypertension. The European Paediatric Pulmonary Vascular Disease Network, endorsed by ISHLT and DGPK. Heart 2016;102 Suppl 2:ii23-9.

6. Ploegstra MJ, Zijlstra WM, Douwes JM, et al. Prognostic factors in pediatric pulmonary arterial hypertension: A systematic review and meta-analysis. Int J Cardiol 2015;184:198-207.

7. van Loon RL, Roofthooft MT, Delhaas T, et al. Outcome of pediatric patients with pulmonary arterial hypertension in the era of new medical therapies. Am J Cardiol 2010;106:117-24.

8. Ivy DD, Abman SH, Barst RJ, et al. Pediatric pulmonary hypertension. J Am Coll Cardiol 2013;62:D117-26.

9. Zijlstra WMH, Douwes JM, Rosenzweig EB, et al. Survival differences in pediatric pulmonary arterial hypertension: clues to a better understanding of outcome and optimal treatment strategies. J Am Coll Cardiol 2014;63:2159-69.

10. Naeije R, Vachiery JL, Yerly P, et al. The transpulmonary pressure gradient for the diagnosis of pulmonary vascular disease. Eur Respir J 2013;41:217-23.

11. Gerges C, Gerges M, Lang MB, et al. Diastolic pulmonary vascular pressure gradient: a predictor of prognosis in "out-of-proportion" pulmonary hypertension. Chest 2013;143:758-766.

12. Mazimba S, Mejia-Lopez E, Black G, et al. Diastolic pulmonary gradient predicts outcomes in group 1 pulmonary hypertension (analysis of the NIH primary pulmonary hypertension registry). Respir Med 2016;119:81-6.

13. LaFarge CG, Miettinen OS. The estimation of oxygen consumption. Cardiovasc Res 1970;4:23-30.

14. Sitbon O, Humbert M, Jaïs $X$, et al. Long-term response to calcium channel blockers in idiopathic pulmonary arterial hypertension. Circulation 2005;111:3105-11.

15. Douwes JM, Humpl T, Bonnet D, et al. Acute vasodilator response in pediatric pulmonary arterial hypertension: current clinical practice from the TOPP registry. J Am Coll Cardiol 2016;67:1312-23. 
16. Douwes JM, Roofthooft MT, Bartelds B, et al. Pulsatile haemodynamic parameters are predictors of survival in paediatric pulmonary arterial hypertension. Int J Cardiol 2013;168:1370-7.

17. Takatsuki S, Nakayama T, Ikehara S, et al. Pulmonary Arterial Capacitance Index Is a Strong Predictor for Adverse Outcome in Children with Idiopathic and Heritable Pulmonary Arterial Hypertension. J Pediatr 2017;180:75-9.e2.

18. Apitz C, Zimmermann R, Kreuder J, et al. Assessment of pulmonary endothelial function during invasive testing in children and adolescents with idiopathic pulmonary arterial hypertension. J Am Coll Cardiol 2012;60:157-64.

19. Benza RL, Miller DP, Gomberg-Maitland M, et al.

Cite this article as: Apitz C, Berger RMF, Ivy DD, Humpl T, Bonnet D, Beghetti M, Schranz D, Latus H; on behalf of the TOPP investigators. Hemodynamic and prognostic impact of the diastolic pulmonary arterial pressure in children with pulmonary arterial hypertension-a registry-based analysis. Cardiovasc Diagn Ther 2021;11(4):1037-1047. doi: 10.21037/ cdt-20-934
Predicting survival in pulmonary arterial hypertension: insights from the Registry to Evaluate Early and LongTerm Pulmonary Arterial Hypertension Disease Management (REVEAL). Circulation 2010;122:164-72.

20. Barst RJ, McGoon MD, Elliott CG, et al. Survival in childhood pulmonary arterial hypertension: insights from the registry to evaluate early and long-term pulmonary arterial hypertension disease management. Circulation 2012;125:113-22.

21. Rich S, Kaufmann E. High dose titration of calcium channel blocking agents for primary pulmonary hypertension: guidelines for short-term drug testing. J Am Coll Cardiol 1991;18:1323-7. 